\title{
Correlation of the neutron star crust-core properties with the slope of the symmetry energy and the lead skin thickness
}

\author{
H. Pais, ${ }^{1}$ A. Sulaksono, ${ }^{2}$ B. K. Agrawal, ${ }^{3}$ and C. Providência ${ }^{1}$ \\ ${ }^{1}$ CFisUC, Department of Physics, University of Coimbra, 3004-516 Coimbra, Portugal \\ ${ }^{2}$ Departemen Fisika, FMIPA, Universitas Indonesia, Depok 16424, Indonesia \\ ${ }^{3}$ Saha Institute of Nuclear Physics, Kolkata 700064, India \\ (Received 7 January 2016; revised manuscript received 16 February 2016; published 7 April 2016)
}

\begin{abstract}
The correlations of the crust-core transition density and pressure in neutron stars with the slope of the symmetry energy and the neutron skin thickness are investigated, using different families of relativistic mean-field parametrizations with constant couplings and nonlinear terms mixing the $\sigma-, \omega$-, and $\rho$-meson fields. It is shown that the modification of the density dependence of the symmetry energy, involving the $\sigma$ or the $\omega$ meson, gives rise to different behaviors: the effect of the $\omega$ meson may also be reproduced within nonrelativistic phenomenological models, while the effect of the $\sigma$ meson is essentially relativistic. Depending on the parametrization with $\sigma-\rho$ or $\omega-\rho$ mixing terms, different values of the slope of the symmetry energy at saturation must be considered in order to obtain a neutron matter equation of state compatible with results from chiral effective field theory. This difference leads to different pressures at the crust-core transition density. A linear correlation between the transition density and the symmetry energy slope or the neutron skin thickness of the ${ }^{208} \mathrm{~Pb}$ nucleus is obtained, only when the $\omega$ meson is used to describe the density dependence of the symmetry energy. A comparison is made between the crust-core transition properties of neutron stars obtained by three different methods, the relativistic random phase approximation (RRPA), the Vlasov equation, and thermodynamical method. It is shown that the RRPA and the Vlasov methods predict similar transition densities for $p n e \quad \beta$-equilibrium stellar matter.
\end{abstract}

DOI: 10.1103/PhysRevC.93.045802

\section{INTRODUCTION}

Neutron stars (NSs), with their extreme properties like very high densities and pressures, are an obvious laboratory to study nuclear physics, as they are a window into the microscopic properties of nuclear matter at extreme isospin asymmetries [1]. The properties of asymmetric nuclear systems have also been studied in terrestrial laboratories in past years [2,3], but many aspects still rely on theoretical models. Constraints on the behavior of the symmetry energy above nuclear saturation density have been coming from experiments with new neutron-rich radioactive beams, and in relativistic heavy-ion collisions, giant monopole resonances [4], isobaric analog states [5], or meson production (pions [6] and kaons [7]) in heavy-ion collisions.

Correlations between different quantities in the bulk matter and finite nuclei were established, like the correlation between the pressure of neutron matter at $\rho=0.1 \mathrm{fm}^{-3}$ and the neutron skin thickness of ${ }^{208} \mathrm{~Pb}[8,9]$, the correlation between the crust-core transition density and the neutron skin thickness of ${ }^{208} \mathrm{~Pb}$ [10], and the correlation between the slope $L$ and the curvature $K_{\text {sym }}$ of the symmetry energy with the neutron skin thickness and the crust-core transition density in compact stars [11]. These correlations, together with terrestrial and observational constraints, will allow the construction of appropriate equations of state (EOS).

In Ref. [12], it was shown that the glitches of Vela, which are thought to occur due to angular momentum transfer between the crust and the core, could be explained if at least $1.4 \%$ of the total moment of inertia of the star resides in the inner crust. Moreover, the same authors also showed that the crustal moment of inertia is sensitive to the pressure at the crust-core interface. Later, this mechanism was questioned because neutron entrainment would require that the inner crust contributes at least $7 \%$ of the total star moment of inertia [13]. Entrainment seems to indicate that the crust is not enough to account for the observed glitches [14]. Recently, however, it was argued that uncertainties on the crust EOS are still large and the mechanism of glitches may be totally explained by the crust if an appropriate EOS is considered, e.g., an EOS that predicts a large transition pressure [15]. This was possible by employing a family of EOS where the density dependence of the symmetry energy was accounted for, including in the Lagrangian density a term that mixes the $\rho$ and the $\omega$ mesons.

Nonlinear meson terms have been included in the Lagrangian formulation of relativistic mean-field (RMF) models in order to modify the density dependence of the EOS, both isoscalar and isovector channels $[10,16]$. A different approach was considered in Ref. [17], where nonlinear terms were avoided at the expense of the inclusion of density dependent couplings in the Lagrangian density. In Ref. [18], the density dependence of the symmetry energy was described within an extended RMF model, including both self-interaction and mixed interaction terms involving the scalar-isoscalar, vectorisoscalar, and vector-isovector mesons up to the quartic order. The parameters of the models were fitted to nuclear properties and the neutron thickness of the ${ }^{208} \mathrm{~Pb}$ was allowed to vary in the range $\sim 0.20-0.24 \mathrm{fm}$.

An expansion of the energy density of a system of nucleons described within RMF models in powers of the Fermi momentum shows that the $\sigma$ meson plays a special role in RMF models, giving rise to terms similar to many-body repulsive terms in nonrelativistic models $[19,20]$. In fact, saturation is attained in RMF models due to the quenching of the $\sigma$ 
meson with density, whereas nonrelativistic models have to introduce three-body repulsive interactions in order to describe saturation correctly. In Ref. [21], the high density EOS of nuclear matter was modified using a mixed $\sigma-\omega$ term, and it has been shown that improvements were attained in the description of the binding energy systematics and the EOS for the dilute neutron matter, with respect to a simple quartic $\omega$ term. In the present study, we investigate the effect of modifying the density dependence of the symmetry energy using a mixed $\sigma-\rho$ term, instead of a $\omega-\rho$ term as in Ref. [15]. $\sigma-\rho$ mixed terms were first included in Ref. [10], where a quartic term was introduced in the Lagrangian density. In our study we consider instead a third order term, $\sim \sigma \rho^{2}$.

In this work, we investigate the correlation between the transition density and pressure from the inner crust to the core of neutron stars. We use three different methods, the RRPA method [22], the Vlasov formalism [23], and the thermodynamical method [21,24-26], at zero temperature and for $\beta$-equilibrium matter. We also investigate the effect of the contribution of the electrons, the Coulomb interaction, and the nonlinear $\omega-\rho$ coupling term. The paper is organized as follows. In Secs. II and III, we introduce the formalism used in this study; in Sec. IV, we present and discuss the results obtained; and finally, in Sec. V, some conclusions are drawn.

\section{MODEL FOR NEUTRON STAR MATTER}

We use the relativistic nonlinear Walecka model (NLWM) [27] in the mean-field approximation to study asymmetric nuclear and stellar matter at zero temperature. We consider a system of baryons with mass $M$, interacting with and through an isoscalar-scalar field $\sigma$ with mass $m_{\sigma}$, an isoscalar-vector field $\omega^{\mu}$ with mass $m_{\omega}$, and an isovectorvector field $\rho^{\mu}$ with mass $m_{\rho}$. When describing npe matter, we also include a system of electrons with mass $m_{e}$. Protons and electrons interact through the electromagnetic field $A^{\mu}$. The Lagrangian density reads [21]

$$
\mathcal{L}=\mathcal{L}_{N M}+\mathcal{L}_{e}+\mathcal{L}_{\sigma}+\mathcal{L}_{\omega}+\mathcal{L}_{\rho}+\mathcal{L}_{\sigma \omega \rho}+\mathcal{L}_{A} .
$$

Here, the Lagrangian $\mathcal{L}_{N M}$ describes the linear interactions of the nucleons through the mesons exchange. The explicit form of $\mathcal{L}_{N M}$ is

$$
\begin{aligned}
\mathcal{L}_{N M}= & \sum_{N=n, p} \bar{\Psi}_{N}\left[i \gamma^{\mu} \partial_{\mu}-\left(M-g_{\sigma} \sigma\right)\right. \\
& \left.-\left(g_{\omega} \gamma^{\mu} \omega_{\mu}+\frac{1}{2} g_{\rho} \gamma^{\mu} \boldsymbol{\tau} \cdot \rho_{\mu}\right)\right] \Psi_{N},
\end{aligned}
$$

where the sum is taken over the neutrons and protons, and $\boldsymbol{\tau}$ are the Pauli matrices. The electron Lagrangian is given by

$$
\mathcal{L}_{e}=\bar{\psi}_{e}\left[\gamma_{\mu}\left(i \partial^{\mu}+e A^{\mu}\right)-m_{e}\right] \psi_{e}
$$

and

$$
\mathcal{L}_{A}=-\frac{1}{4} F_{\mu \nu} F^{\mu \nu}
$$

with $F_{\mu \nu}=\partial_{\mu} A_{\nu}-\partial_{\nu} A_{\mu}$. The Lagrangian densities describing the free mesons and self-interactions for $\sigma, \omega$, and $\rho$ mesons, respectively, can be written as

$$
\begin{aligned}
& \mathcal{L}_{\sigma}=\frac{1}{2}\left(\partial_{\mu} \sigma \partial^{\mu} \sigma-m_{\sigma}^{2} \sigma^{2}\right)-\frac{\kappa_{3}}{6 M} g_{\sigma} m_{\sigma}^{2} \sigma^{3}-\frac{\kappa_{4}}{24 M^{2}} g_{\sigma}^{2} m_{\sigma}^{2} \sigma^{4}, \\
& \mathcal{L}_{\omega}=-\frac{1}{4} \omega_{\mu \nu} \omega^{\mu \nu}+\frac{1}{2} m_{\omega}^{2} \omega_{\mu} \omega^{\mu}+\frac{1}{24} \zeta_{0} g_{\omega}^{2}\left(\omega_{\mu} \omega^{\mu}\right)^{2} \\
& \mathcal{L}_{\rho}=-\frac{1}{4} \rho_{\mu \nu} \cdot \rho^{\mu \nu}+\frac{1}{2} m_{\rho}^{2} \rho_{\mu} \cdot \boldsymbol{\rho}^{\mu}
\end{aligned}
$$

The $\omega^{\mu \nu}$ and $\rho^{\mu \nu}$ are antisymmetric field tensors corresponding to the $\omega$ and $\rho$ mesons. They are defined as $\omega^{\mu \nu}=\partial^{\mu} \omega^{\nu}-$ $\partial^{v} \omega^{\mu}$ and $\boldsymbol{\rho}^{\mu \nu}=\partial^{\mu} \boldsymbol{\rho}^{v}-\partial^{v} \boldsymbol{\rho}^{\mu}-g_{\rho}\left(\boldsymbol{\rho}^{\mu} \times \boldsymbol{\rho}^{\nu}\right)$. The mixing nonlinear $\sigma, \omega$, and $\rho$ mesons are described by $\mathcal{L}_{\sigma \omega \rho}$ :

$$
\begin{aligned}
\mathcal{L}_{\sigma \omega \rho}= & \frac{\eta_{1}}{2 M} g_{\sigma} m_{\omega}^{2} \sigma \omega_{\mu} \omega^{\mu}+\frac{\eta_{2}}{4 M^{2}} g_{\sigma}^{2} m_{\omega}^{2} \sigma^{2} \omega_{\mu} \omega^{\mu} \\
& +\frac{\eta_{\rho}}{2 M} g_{\sigma} m_{\rho}^{2} \sigma \boldsymbol{\rho}_{\mu} \cdot \boldsymbol{\rho}^{\mu}+\frac{\eta_{1 \rho}}{4 M^{2}} g_{\sigma}^{2} m_{\rho}^{2} \sigma^{2} \boldsymbol{\rho}_{\mu} \cdot \boldsymbol{\rho}^{\mu} \\
& +\frac{\eta_{2 \rho}}{4 M^{2}} g_{\omega}^{2} m_{\rho}^{2} \omega_{\mu} \omega^{\mu} \boldsymbol{\rho}_{\mu} \cdot \boldsymbol{\rho}^{\mu}
\end{aligned}
$$

Of particular interest in the present work are the crosscoupling terms involving the $\rho$-meson field, which contributes to the isovector part of the effective Lagrangian density, in addition to the usual linear couplings of the $\rho$ meson to the nucleons. We mainly focus on the lowest order $\sigma-\rho$ and $\omega-\rho$ cross couplings whose strengths are determined by the values of $\eta_{\rho}$ and $\eta_{2 \rho}$. The quartic order $\sigma$ - $\rho$ cross-coupling strength $\eta_{1 \rho}$ is set to zero. The values of $\eta_{\rho}$ or $\eta_{2 \rho}$ can be appropriately adjusted to yield wide variations in the density dependence of the symmetry energy coefficient and the neutron skin thickness in heavy nuclei without affecting the other properties of finite nuclei [28-30].

To study the role of the $\sigma-\rho$ and $\omega-\rho$ mixing terms on the crust-core transition properties in neutron stars, we use two different families of RMF model, $F_{\rho}$ and $F_{2 \rho}[18,31]$. The isovector part of the Lagrangian density for the $F_{\rho}\left(F_{2 \rho}\right)$ family is governed by the couplings $g_{\rho}$ and $\eta_{\rho}\left(\eta_{2 \rho}\right)$. The coupling $\eta_{1 \rho}$ is set to zero for both the families. The different parametrizations of $F_{\rho}\left(F_{2 \rho}\right)$ families are obtained by varying appropriately the values of $g_{\rho}$ and $\eta_{\rho}\left(\eta_{2 \rho}\right)$. For a given value of $\eta_{\rho}\left(\eta_{2 \rho}\right)$, the value of $g_{\rho}$ is always adjusted to yield appropriate binding energy for the ${ }^{208} \mathrm{~Pb}$ nucleus. Once the values of $g_{\rho}$ and $\eta_{\rho}$ or $\eta_{2 \rho}$ are known, the properties of the nuclear matter and the finite nuclei can be computed. The values of $\eta_{\rho}$ and $\eta_{2 \rho}$ are varied in the ranges $0-12$ and $0-60$, respectively.

In Table I, we present the parameters that differ among the models as considered in the present work. The remaining parameters, which correspond to the isoscalar part of the Lagrangian density, and the masses of the $\sigma, \omega$, and $\rho$ mesons are kept fixed to those for the BKA22 model [18]. The values of these parameters are $g_{\sigma} /(4 \pi)=0.8462, g_{\omega} /(4 \pi)=1.1089$, $k_{3}=1.55, k_{4}=2.13451, \zeta_{0}=5.8253, \eta_{1}=0.1555, \eta_{2}=$ 0.0697 , and $\eta_{1 \rho}=0$. The masses for the $\omega, \rho$, and $\sigma$ mesons are $m_{\omega}=782.5 \mathrm{MeV}, m_{\rho}=770 \mathrm{MeV}$, and $m_{\sigma}=497.8578 \mathrm{MeV}$. The nucleon mass is set to $939 \mathrm{MeV}$. Table I also shows the saturation properties, the neutron skin thickness of ${ }^{208} \mathrm{~Pb}$, and the crust-core transition densities, $\rho_{\text {trans }}$, calculated within 
TABLE I. The coupling parameters $g_{\rho}$ and $\eta_{\rho}\left(\eta_{2 \rho}\right)$ for the $F_{\rho}\left(F_{2 \rho}\right)$ families, and the corresponding values for the symmetry energy coefficient, $E_{\text {sym }}$, and the symmetry energy slope parameter $L$ (in $\mathrm{MeV}$ ), evaluated at the saturation density, $\rho_{0}=0.148 \mathrm{fm}^{-3}$. The values of the neutron skin thickness, $\Delta r_{\mathrm{np}}$ (in fm), for the ${ }^{208} \mathrm{~Pb}$ nucleus, and the crust-core transition densities, $\rho_{\text {trans }}\left(\mathrm{in} \mathrm{fm}^{-3}\right)$, calculated within the Vlasov formalism, for $\beta$-equilibrium pne matter at $T=0 \mathrm{MeV}$, for all the models are also listed. The binding energy, $E / A$, is $-16.08 \mathrm{MeV}$, the incompressibility $K$ is $228.63 \mathrm{MeV}$, and the skewness coefficient $Q_{0}$ is $-285.03 \mathrm{MeV}$.

\begin{tabular}{lrrrrrc}
\hline \hline Model & \multicolumn{1}{c}{$g_{\rho}$} & $\eta_{\rho}$ & $E_{\text {sym }}$ & \multicolumn{1}{c}{$L$} & $\Delta r_{\text {np }}$ & $\rho_{\text {trans }}$ \\
\hline$F_{\rho}-1$ & 8.8614 & 0 & 36.4 & 108.9 & 0.280 & 0.0489 \\
$F_{\rho}-2$ & 11.1799 & 2.0 & 34.3 & 86.9 & 0.241 & 0.0570 \\
$F_{\rho}-3$ & 13.0335 & 4.0 & 33.4 & 79.1 & 0.219 & 0.0589 \\
$F_{\rho}-4$ & 14.6294 & 6.0 & 32.8 & 75.2 & 0.206 & 0.0591 \\
$F_{\rho}-5$ & 16.0494 & 8.0 & 32.4 & 72.8 & 0.194 & 0.0588 \\
$F_{\rho}-6$ & 17.3450 & 10.0 & 32.1 & 71.3 & 0.185 & 0.0585 \\
$F_{\rho}-7$ & 18.5388 & 12.0 & 31.9 & 70.1 & 0.179 & 0.0582 \\
Model & $g_{\rho}$ & $\eta_{2 \rho}$ & $E_{\text {sym }}$ & $L$ & $\Delta r_{\text {np }}$ & $\rho_{\text {trans }}$ \\
\hline$F_{2 \rho}-1$ & 9.2225 & 2.5 & 35.6 & 97.3 & 0.269 & 0.0525 \\
$F_{2 \rho}-2$ & 9.5656 & 5.0 & 35.0 & 88.5 & 0.259 & 0.0566 \\
$F_{2 \rho}-3$ & 9.8898 & 7.5 & 34.5 & 81.7 & 0.250 & 0.0610 \\
$F_{2 \rho}-4$ & 10.1964 & 10.0 & 34.1 & 76.2 & 0.242 & 0.0652 \\
$F_{2 \rho}-5$ & 11.3135 & 20.0 & 32.8 & 62.5 & 0.215 & 0.0759 \\
$F_{2 \rho}-6$ & 13.1771 & 40.0 & 31.3 & 50.9 & 0.178 & 0.0807 \\
$F_{2 \rho}-7$ & 14.7328 & 60.0 & 30.4 & 46.0 & 0.152 & 0.0805 \\
\hline \hline
\end{tabular}

the Vlasov formalism, for $\beta$-equilibrium pne matter at $T=$ $0 \mathrm{MeV}$, for all the models considered in the present work.

\section{METHODS TO CALCULATE NS CRUST-CORE PROPERTIES}

In the present section, we review the three methods that are used to determine the crust-core transition density: the thermodynamical method, the dynamical spinodal from the Vlasov equation, and the relativistic random phase approximation (RRPA) formalism.

\section{A. Thermodynamical method}

For a system to be stable against small density fluctuations, the thermodynamical method requires that the conditions of mechanical and chemical stabilities should be satisfied [32-34]; i.e.,

$$
\begin{aligned}
& -\left(\frac{\partial P}{\partial v}\right)_{\hat{\mu}}>0, \\
& -\left(\frac{\partial \hat{\mu}}{\partial q_{c}}\right)_{v}>0 .
\end{aligned}
$$

Here $P=P_{b}+P_{e}$ is the total pressure of the neutron, proton, and electron system, with the contributions $P_{b}$ and $P_{e}$ from baryons and electrons, respectively. The $v$ and $q_{c}$ are the volume and charge per baryon number. The $\hat{\mu}$ is the chemical potential defined as $\hat{\mu}=\mu_{n}-\mu_{p}$. Since the system under consideration is in $\beta$ equilibrium, which implies $\hat{\mu}=\mu_{e}$, the electron pressure $P_{e}$ is a function of $\hat{\mu}$. Equation (8) becomes

$$
-\left(\frac{\partial P_{b}}{\partial v}\right)_{\hat{\mu}}>0 .
$$

Equations (9) and (10) can be expressed in terms of the energy per nucleon, $E_{b}\left(\rho, x_{p}\right)$, at a given density $\rho$ and proton fraction $x_{p}$ as

$$
\begin{aligned}
-\left(\frac{\partial q_{c}}{\partial \hat{\mu}}\right)_{v}= & \left(\frac{\partial^{2} E_{b}\left(\rho, x_{p}\right)}{\partial x_{p}^{2}}\right)^{-1}+\frac{\mu_{e}^{2}}{\pi^{2} \hbar^{3} \rho} \\
-\left(\frac{\partial P_{b}}{\partial v}\right)_{\hat{\mu}}= & \rho^{2}\left[2 \rho \frac{\partial E_{b}\left(\rho, x_{p}\right)}{\partial \rho}+\rho^{2} \frac{\partial^{2} E_{b}\left(\rho, x_{p}\right)}{\partial^{2} \rho}\right. \\
& \left.-\frac{\frac{\partial^{2} E_{b}\left(\rho, x_{p}\right) \rho^{2}}{\partial \rho \partial x_{p}}}{\frac{\partial^{2} E_{b}\left(\rho, x_{p}\right)}{\partial x_{p}^{2}}}\right]>0 .
\end{aligned}
$$

Equation (11) is usually valid; thus, the crust-core transition density is determined by using the inequality of Eq. (12).

\section{B. Vlasov method}

In Refs. [35,36], the collective modes in cold nuclear matter were determined within the Vlasov equation, based on the Walecka model [27], and later also used in Refs. [20,37]. We extended this formalism to include the mixing nonlinear selfinteractions of the mesons $\sigma, \omega$, and $\rho$ in Ref. [38].

The time evolution of the distribution functions $f_{i}$ is described by the Vlasov equation, which expresses the conservation of the number of particles in phase space and is, therefore, covariant. At zero temperature, the state that minimizes the energy of asymmetric nuclear matter is characterized by the Fermi momenta $P_{F i}, i=p, n, P_{F e}=P_{F p}$, and is described by the distribution function

$$
f_{0}(\boldsymbol{r}, \boldsymbol{p})=\operatorname{diag}\left[\Theta\left(P_{F p}^{2}-p^{2}\right), \Theta\left(P_{F n}^{2}-p^{2}\right), \Theta\left(P_{F e}^{2}-p^{2}\right)\right]
$$

and by the constant mesonic fields.

Collective modes in the present approach correspond to small oscillations around the equilibrium state. These small deviations are described by the linearized equations of motion and collective modes are given as solutions of those equations.

The linearized Vlasov equations for $\delta f_{i}$,

$$
\frac{d \delta f_{i}}{d t}+\left\{\delta f_{i}, h_{0 i}\right\}+\left\{f_{0 i}, \delta h_{i}\right\}=0,
$$

are equivalent to the following time-evolution equations:

$$
\begin{aligned}
\frac{\partial S_{i}}{\partial t}+\left\{S_{i}, h_{0 i}\right\} & =\delta h_{i}=-g_{\sigma} \frac{M^{*}}{\epsilon_{0}} \delta \phi-\frac{\mathbf{p} \cdot \delta \mathcal{V}_{i}}{\epsilon_{0}}+\delta \mathcal{V}_{0 i} \\
i & =p, n \\
\frac{\partial S_{e}}{\partial t}+\left\{S_{e}, h_{0 e}\right\} & =\delta h_{e}=-e\left[\delta A_{0}-\frac{\mathbf{p} \cdot \delta \mathbf{A}}{\epsilon_{0 e}}\right]
\end{aligned}
$$


where

$$
\begin{aligned}
\delta f_{i} & =\left\{S_{i}, f_{0 i}\right\}, \\
\delta \mathcal{V}_{0 i} & =g_{\omega} \delta \omega_{0}+\tau_{i} \frac{g_{\rho}}{2} \delta \rho_{0}+e \frac{1+\tau_{i}}{2} \delta A_{0}, \\
\delta \mathcal{V}_{i} & =g_{\omega} \delta \boldsymbol{\omega}+\tau_{i} \frac{g_{\rho}}{2} \delta \boldsymbol{\rho}+e \frac{1+\tau_{i}}{2} \delta \mathbf{A}, \\
h_{0 i} & =\epsilon_{0}+\mathcal{V}_{0 i}^{(0)}=\sqrt{p^{2}+M^{* 2}}+\mathcal{V}_{0 i}^{(0)}, \\
h_{0 e} & =\epsilon_{0 e}=\sqrt{p^{2}+m_{e}^{2}},
\end{aligned}
$$

which has only to be satisfied for $p=P_{F i}$.

We are interested in the longitudinal modes, with wave vector $\mathbf{k}$ and frequency $\omega$, which are described by the ansatz

$$
\left(\begin{array}{c}
S_{j}(\mathbf{r}, \mathbf{p}, t) \\
\delta \phi \\
\delta \zeta_{0} \\
\delta \zeta_{i}
\end{array}\right)=\left(\begin{array}{c}
\mathcal{S}_{\omega}^{j}(\cos \theta) \\
\delta \phi_{\omega} \\
\delta \zeta_{\omega}^{0} \\
\delta \zeta_{\omega}^{i}
\end{array}\right) \mathrm{e}^{i(\omega t-\mathbf{k} \cdot \mathbf{r})}
$$

where $j=p, n, e ; \zeta=\omega, \rho, A$ represent the vector-meson fields, and $\theta$ is the angle between $\mathbf{p}$ and $\mathbf{k}$. The wave vector of the excitation mode, $\mathbf{k}$, is identified with the momentum transferred to the system through the process which gives rise to the excitation.

Replacing the ansatz (16) in Eqs. (14) and (15), we get a set of equations for the fields and for the generating functions. The solutions of these equations form a complete set of eigenmodes that may be used to construct a general solution for an arbitrary longitudinal perturbation. They lead to the following matrix equation:

$$
\left(\begin{array}{ccc}
1+F^{p p} L_{p} & F^{p n} L_{p} & C_{A}^{p e} L_{p} \\
F^{\mathrm{np}} L_{n} & 1+F^{n n} L_{n} & 0 \\
C_{A}^{e p} L_{e} & 0 & 1-C_{A}^{e e} L_{e}
\end{array}\right)\left(\begin{array}{c}
A_{\omega p} \\
A_{\omega n} \\
A_{\omega e}
\end{array}\right)=0
$$

with $\quad A_{\omega i}=\int_{-1}^{1} x S_{\omega i}(x) d x, \quad L_{i}=L\left(s_{i}\right)=2-s_{i} \ln \left(\left(s_{i}+\right.\right.$ 1) $\left./\left(s_{i}-1\right)\right)$, where $s_{i}=\omega / \omega_{0 i}$, and $F^{i j}=C_{s}^{i j}-C_{v}^{i j}-$ $C_{\rho}^{i j}-C_{A}^{i j} \delta_{i p} \delta_{i j}$. The coefficients $C_{s}^{i j}, C_{v}^{i j}, C_{\rho}^{i j}$, and $C_{A}^{i j}$ are given in Ref. [38].
At subsaturation densities, there are unstable modes identified by imaginary frequencies. For these modes we define the growth rate $\Gamma=-i \omega$. The region in $\left(\rho_{p}, \rho_{n}\right)$ space for a given wave vector $\mathbf{k}$ and temperature $T$, limited by the surface $\omega=0$, defines the dynamical spinodal surface. In the $k=0 \mathrm{MeV}$ limit, we recover the thermodynamic spinodal, which is defined by the surface in the $\left(\rho_{p}, \rho_{n}, T\right)$ space for which the curvature matrix of the free energy density is zero, i.e., has a zero eigenvalue. This relation was discussed in Ref. [39].

\section{RRPA method}

The longitudinal dielectric function can be written as [40]

$$
\varepsilon_{L}=\operatorname{det}\left[1-D_{L}(q) \Pi_{L}\left(q, q_{0}=0\right)\right] .
$$

The uniform ground state system becomes unstable to smallamplitude density fluctuations with momentum transfer $q$ when $\varepsilon_{L} \leqslant 0$. Note that in Eq. (18), $q_{0}$ is the time component of the four-momentum transfer $q^{\mu}=\left(q_{0}, \vec{q}\right)$ and $q=|\vec{q}|$. The transition density, $\rho_{t}$, is the largest density for which the above condition has a solution. For matter that in general consists of protons, neutrons, electrons, and muons, the longitudinal meson propagator is given by

$$
D_{L}=\left(\begin{array}{ccccc}
d_{g} & d_{g} & 0 & -d_{g} & 0 \\
d_{g} & d_{g} & 0 & -d_{g} & 0 \\
0 & 0 & -d_{s} & d_{s v \rho}^{+} & d_{s v \rho}^{-} \\
-d_{g} & -d_{g} & d_{s v \rho}^{+} & d_{33} & d_{v \rho}^{-} \\
0 & 0 & d_{s v \rho}^{-} & d_{v \rho}^{-} & d_{44}
\end{array}\right),
$$

where $d_{s v \rho}^{+}=-\left(d_{s v}+d_{s \rho}\right), d_{s v \rho}^{-}=-\left(d_{s v}-d_{s \rho}\right), d_{v \rho}^{-}=d_{v}-$ $d_{\rho}, d_{33}=d_{g}+d_{v}+d_{\rho}+2 d_{v \rho}$, and $d_{44}=d_{v}+d_{\rho}-2 d_{v \rho}$. In this form, mixing propagators between isoscalar-scalar and isoscalar-vector $\left(d_{s v}\right)$, isoscalar-vector and isovector-vector $\left(d_{v \rho}\right)$, isoscalar-scalar and isovector-vector $\left(d_{s \rho}\right)$ are present due to the mixing self-interaction nonlinear terms in the RMF model, in addition to the standard $\gamma, \omega, \sigma$, and $\rho$ propagators $\left(d_{g}, d_{v}, d_{s}\right.$, and $\left.d_{\rho}\right)$. These propagators are determined from the quadratic fluctuations around the static solutions which are generated by the second derivatives of energy density $\left(\partial^{2} \epsilon / \partial \phi_{i} \partial \phi_{j}\right)$, where $\phi_{i}$ and $\phi_{j}$ are the involved meson fields. The explicit forms of the $\sigma, \omega$, and $\rho$ propagators are

$$
\begin{aligned}
& d_{s}=\frac{g_{\sigma}^{2}\left(q^{2}+m_{\omega}^{* 2}\right)\left(q^{2}+m_{\rho}^{* 2}\right)}{\left(q^{2}+m_{\omega}^{* 2}\right)\left(q^{2}+m_{\rho}^{* 2}\right)\left(q^{2}+m_{\sigma}^{* 2}\right)+\left(\Pi_{\sigma \omega}^{0}\right)^{2}\left(q^{2}+m_{\rho}^{* 2}\right)+\left(\Pi_{\sigma \rho}^{0}\right)^{2}\left(q^{2}+m_{\omega}^{* 2}\right)}, \\
& d_{v}=\frac{g_{\omega}^{2}\left(q^{2}+m_{\sigma}^{* 2}\right)\left(q^{2}+m_{\rho}^{* 2}\right)}{\left(q^{2}+m_{\omega}^{* 2}\right)\left(q^{2}+m_{\rho}^{* 2}\right)\left(q^{2}+m_{\sigma}^{* 2}\right)+\left(\Pi_{\sigma \omega}^{0}\right)^{2}\left(q^{2}+m_{\rho}^{* 2}\right)-\left(\Pi_{\omega \rho}^{00}\right)^{2}\left(q^{2}+m_{\sigma}^{* 2}\right)}, \\
& d_{\rho}=\frac{1 / 4 g_{\rho}^{2}\left(q^{2}+m_{\sigma}^{* 2}\right)\left(q^{2}+m_{\omega}^{* 2}\right)}{\left(q^{2}+m_{\omega}^{* 2}\right)\left(q^{2}+m_{\rho}^{* 2}\right)\left(q^{2}+m_{\sigma}^{* 2}\right)+\left(\Pi_{\sigma \rho}^{0}\right)^{2}\left(q^{2}+m_{\omega}^{* 2}\right)-\left(\Pi_{\omega \rho}^{00}\right)^{2}\left(q^{2}+m_{\sigma}^{* 2}\right)},
\end{aligned}
$$


and the meson mixing propagators take the form

$$
\begin{gathered}
d_{s v}=\frac{g_{\sigma} g_{\omega} \Pi_{\omega \sigma}^{0}\left(q^{2}+m_{\rho}^{* 2}\right)}{H\left(q, q_{0}=0\right)}, \\
d_{s \rho}=\frac{1 / 2 g_{\rho} g_{\sigma} \Pi_{\sigma \rho}^{0}\left(q^{2}+m_{\omega}^{* 2}\right)}{H\left(q, q_{0}=0\right)}, \\
d_{v \rho}=\frac{1 / 2 g_{\rho} g_{\omega} \Pi_{\omega \rho}^{00}\left(q^{2}+m_{\sigma}^{* 2}\right)}{H\left(q, q_{0}=0\right)},
\end{gathered}
$$

where the explicit form of $H\left(q, q_{0}=0\right)$ can be written as

$$
\begin{aligned}
H\left(q, q_{0}=0\right)= & \left(q^{2}+m_{\omega}^{* 2}\right)\left(q^{2}+m_{\rho}^{* 2}\right)\left(q^{2}+m_{\sigma}^{* 2}\right) \\
& +\left(\Pi_{\sigma \omega}^{0}\right)^{2}\left(q^{2}+m_{\rho}^{* 2}\right)+\left(\Pi_{\sigma \rho}^{0}\right)^{2}\left(q^{2}+m_{\omega}^{* 2}\right) \\
& -\left(\Pi_{\omega \rho}^{00}\right)^{2}\left(q^{2}+m_{\sigma}^{* 2}\right),
\end{aligned}
$$

and the meson effective masses in Eq. (24) are defined as

$$
\begin{aligned}
m_{\sigma}^{* 2}= & \frac{\partial^{2} \epsilon}{\partial^{2} \sigma}=m_{\sigma}^{2}+\frac{g_{\sigma} m_{\sigma}^{2} \kappa_{3}}{M} \sigma+\frac{g_{\sigma}^{2} m_{\sigma}^{2} \kappa_{4}}{2 M^{2}} \sigma^{2} \\
& -\frac{g_{\sigma}^{2} m_{\omega}^{2} \eta_{2}}{2 M^{2}} \omega_{0}^{2}-\frac{g_{\sigma}^{2} m_{\rho}^{2} \eta_{1 \rho}}{2 M^{2}} \rho_{0}^{2}, \\
m_{\omega}^{* 2}= & -\frac{\partial^{2} \epsilon}{\partial^{2} \omega_{0}}=m_{\omega}^{2}+\frac{g_{\sigma} m_{\omega}^{2} \eta_{1}}{M} \sigma+\frac{g_{\sigma}^{2} m_{\omega}^{2} \eta_{2}}{2 M^{2}} \sigma^{2} \\
& +\frac{\zeta_{0} g_{\omega}^{2}}{2} \omega_{0}^{2}+\frac{g_{\omega}^{2} m_{\rho}^{2} \eta_{2 \rho}}{2 M^{2}} \rho_{0}^{2}, \\
m_{\rho}^{* 2}= & -\frac{\partial^{2} \epsilon}{\partial^{2} \rho_{0}}=m_{\rho}^{2}+\frac{g_{\sigma} m_{\rho}^{2} \eta_{\rho}}{M} \sigma+\frac{g_{\sigma}^{2} m_{\rho}^{2} \eta_{1 \rho}}{2 M^{2}} \sigma^{2} \\
& +\frac{g_{\omega}^{2} m_{\rho}^{2} \eta_{2 \rho}}{2 M^{2}} \omega_{0}^{2},
\end{aligned}
$$

while the polarization due to mesons mixing self-interaction nonlinear terms in Eq. (7) (mix polarizations) are

$$
\begin{aligned}
& \Pi_{\sigma \omega}^{0}=-\frac{\partial^{2} \epsilon}{\partial \sigma \partial \omega_{0}}=\frac{g_{\sigma} m_{\omega}^{2} \eta_{1}}{M} \omega_{0}+\frac{g_{\sigma}^{2} m_{\omega}^{2} \eta_{2}}{M^{2}} \sigma \omega_{0}, \\
& \Pi_{\sigma \rho}^{0}=-\frac{\partial^{2} \epsilon}{\partial \sigma \partial \rho_{0}}=\frac{g_{\sigma} m_{\rho}^{2} \eta_{\rho}}{M} \rho_{0}+\frac{g_{\sigma}^{2} m_{\rho}^{2} \eta_{1 \rho}}{M^{2}} \sigma \rho_{0}, \\
& \Pi_{\omega \rho}^{00}=\frac{\partial^{2} \epsilon}{\partial \omega_{0} \partial \rho_{0}}=-\frac{g_{\omega}^{2} m_{\rho}^{2} \eta_{2 \rho}}{M^{2}} \omega_{0} \rho_{0},
\end{aligned}
$$

whereas the photon propagator takes a standard form, i.e.,

$$
d_{g}=\frac{e^{2}}{q^{2}} .
$$

The longitudinal polarization matrix given in Eq. (18) reads

$$
\Pi_{L}=\left(\begin{array}{ccccc}
\Pi_{00}^{e} & 0 & 0 & 0 & 0 \\
0 & \Pi_{00}^{\mu} & 0 & 0 & 0 \\
0 & 0 & \Pi_{s} & \Pi_{m}^{p} & \Pi_{m}^{n} \\
0 & 0 & \Pi_{m}^{p} & \Pi_{00}^{p} & 0 \\
0 & 0 & \Pi_{m}^{n} & 0 & \Pi_{00}^{n}
\end{array}\right) .
$$

The formulas for polarization elements in $\Pi_{L}$ are given in, e.g., Ref. [22].

\begin{tabular}{|c|c|c|c|c|c|c|}
\hline & & Expt. & $F_{\rho}-3$ & $F_{\rho}-7$ & $F_{2 \rho}-5$ & $F_{2 \rho}-6$ \\
\hline \multirow[t]{4}{*}{${ }^{48} \mathrm{Ca}$} & $E$ & -416.00 & -415.75 & -415.10 & -415.71 & -415.04 \\
\hline & $r_{c}$ & 3.477 & 3.468 & 3.478 & 3.466 & 3.473 \\
\hline & $r_{n}$ & & 3.575 & 3.561 & 3.571 & 3.559 \\
\hline & $\Delta r_{\mathrm{np}}$ & & 0.201 & 0.178 & 0.199 & 0.179 \\
\hline \multirow[t]{4}{*}{${ }^{132} \mathrm{Sn}$} & $E$ & 1102.84 & -1102.49 & -1101.01 & -1102.49 & -1101.07 \\
\hline & $r_{c}$ & 4.709 & 4.736 & 4.751 & 4.728 & 4.739 \\
\hline & $r_{n}$ & & 4.952 & 4.924 & 4.939 & 4.911 \\
\hline & $\Delta r_{\mathrm{np}}$ & & 0.284 & 0.240 & 0.279 & 0.239 \\
\hline \multirow[t]{4}{*}{${ }^{208} \mathrm{~Pb}$} & $E$ & -1636.43 & -1637.07 & -1637.07 & -1637.09 & -1637.03 \\
\hline & $r_{c}$ & 5.501 & 5.545 & 5.559 & 5.537 & 5.547 \\
\hline & $r_{n}$ & & 5.706 & 5.680 & 5.694 & 5.666 \\
\hline & $\Delta r_{\mathrm{np}}$ & & 0.219 & 0.178 & 0.215 & 0.178 \\
\hline
\end{tabular}

For the families of models under study in the present article, the $\Pi_{\sigma \omega}^{0}$ contribution is present, but for the $F_{2 \rho}$ family, the
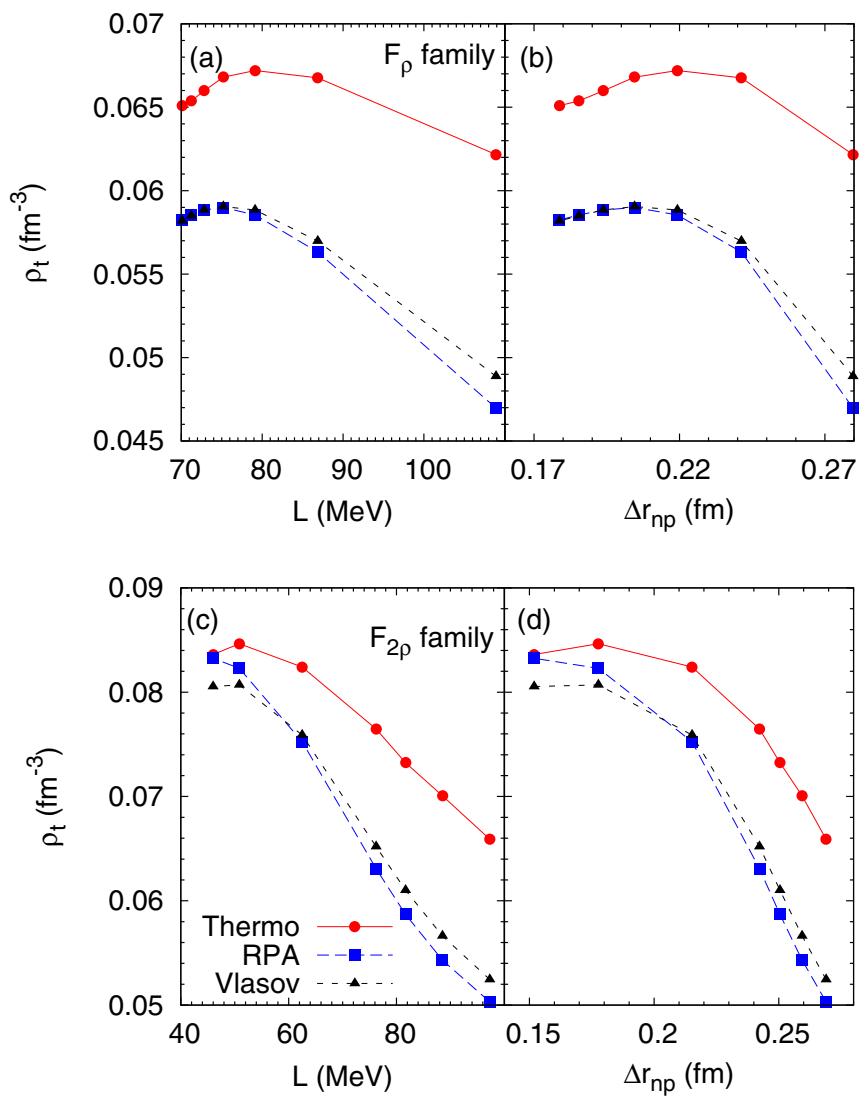

FIG. 1. Crust-core transition density, $\rho_{t}$, for the (a) and (b) $F_{\rho}$ and (c) and (d) $F_{2 \rho}$ families as a function of $L$ (left) and $\Delta r_{\text {np }}$ (right) from RRPA, Vlasov, and thermodynamical methods.

contribution from $\Pi_{\sigma \rho}^{0}$ is zero, and for the $F_{\rho}$ family, the $\Pi_{\omega \rho}^{0}$ contribution becomes zero. In the crust-core region, usually the muons have not yet appeared, so $\Pi_{00}^{\mu}$ can be set to zero, and if we consider the case without electrons, then $\Pi_{00}^{e}$ is also set to zero.

TABLE II. The values of the total binding energy $(E)$ in $\mathrm{MeV}$, charge radii $\left(r_{c}\right)$, neutron radii $\left(r_{n}\right)$, and $\Delta r_{\text {np }}$ (in fm) for a few asymmetric spherical nuclei obtained for selected parametrizations of the $F_{\rho}$ and $F_{2 \rho}$ families. 

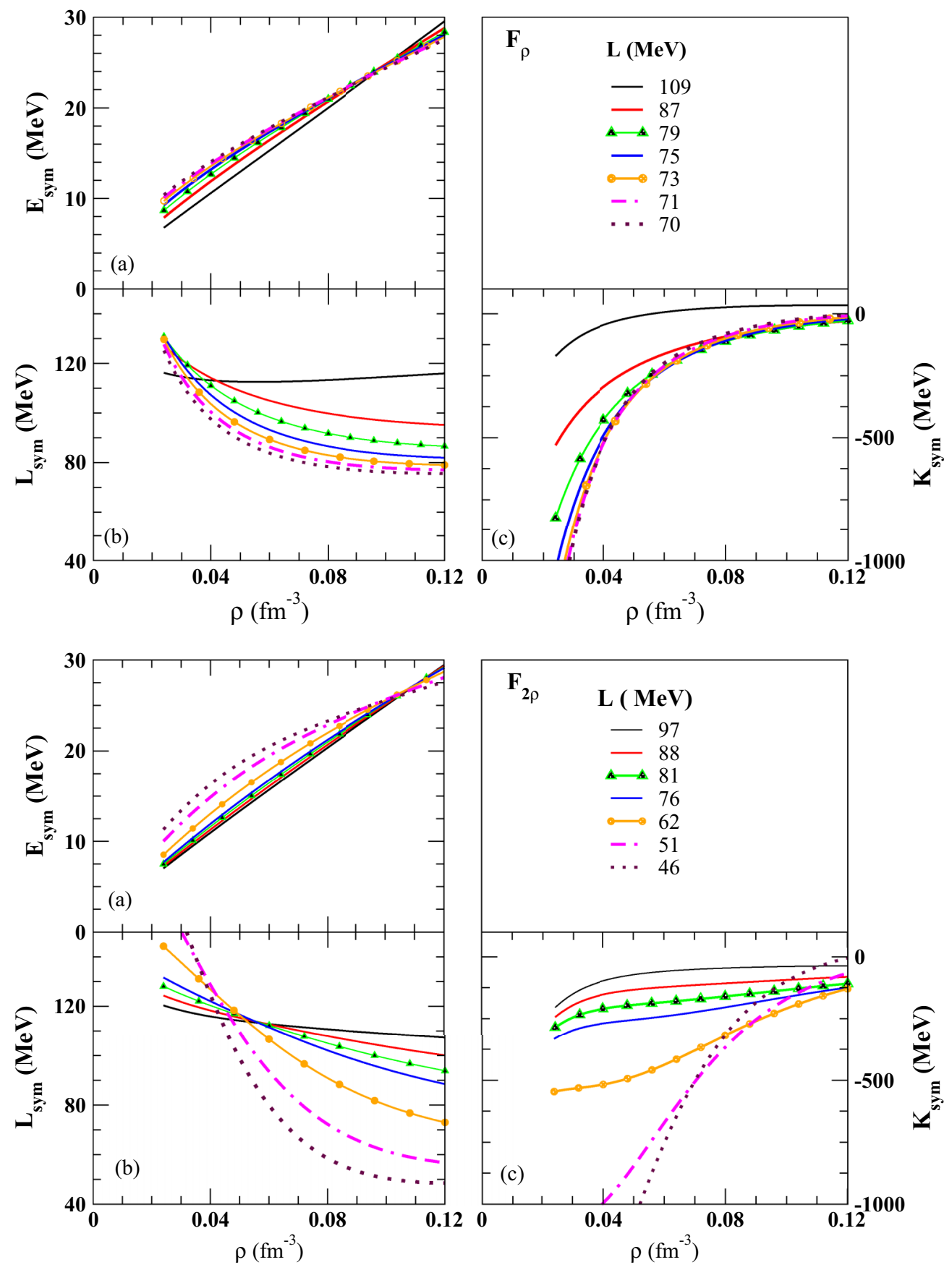

FIG. 2. (a) Symmetry energy $E_{\text {sym }}$, (b) its slope $L$, and (c) curvature $K_{\text {sym }}$, at subsaturation densities for the $F_{\rho}$ (top) and $F_{2 \rho}$ families (bottom).

\section{RESULTS AND DISCUSSION}

In Fig. 1, we plot the crust-core transition density, $\rho_{t}$, as a function of the slope of the symmetry energy $L$ [Figs. 1(a) and 1(c)], and the neutron skin thickness $\Delta r_{\text {np }}$ [Figs. 1(b) and $1(\mathrm{~d})$ ] for the $F_{\rho}$ family (top panel) and for the $F_{2 \rho}$ family (bottom panel). The slope of the symmetry energy is defined as $L=3 \rho_{0}\left(\partial E_{\text {sym }} / \partial \rho\right)_{\rho=\rho_{0}}$; see, e.g., Ref. [11]. The values of $\rho_{t}$ are calculated for the three different methods: RRPA, Vlasov, and thermodynamical. We can see that the transition densities calculated from both the RRPA and Vlasov approaches agree quite well with each other for both families. These results are, therefore, compatible with previous studies executed within Skyrme interactions [41] or RMF models [42], where RRPA calculations and semiclassical calculations were compared.

Comparing the behavior of both families, the $F_{2 \rho}$ family shows, overall, higher transition densities to uniform matter than the $F_{\rho}$ family. The correlation between $\rho_{t}$ and $L$ (or $\Delta r_{\mathrm{np}}$ ) depends significantly on the kind of meson self-interaction mixing terms used in the RMF model. The $\rho_{t}$ evolution predicted by the $F_{\rho}$ family shows a linear correlation with 
$L$ and the neutron thickness in the low $L$ (or $\Delta r_{\mathrm{np}}$ ) region. This behavior differs from the one of the $F_{2 \rho}$ family, where the transition density always decreases with increasing $L$ (or $\left.\Delta r_{\mathrm{np}}\right)$, and from previous works [8-11,25,26].

The density dependence of the symmetry energy is achieved through the inclusion of a mixed term of the $\rho$ meson with the $\sigma$ meson for $F_{\rho}$ and the $\omega$ meson for the $F_{2 \rho}$ families. At subsaturation, the behavior of these two mesons, or, correspondingly, the scalar and the nucleonic densities, are quite different; in particular, the $\sigma$ field, which is responsible for binding the matter, is stronger and increases much faster with density for the values close to zero and slower above $\sim 0.08 \mathrm{fm}^{-3}$. The families $F_{\rho}$ and $F_{2 \rho}$ were built by fitting the binding energy for the ${ }^{208} \mathrm{~Pb}$ nucleus for different values of the neutron skin thickness. Because of the different behavior of the $\sigma$ and $\omega$ mesons, the first one determining the behavior of the $F_{\rho}$ family and the second one the behavior of the $F_{2 \rho}$ family, for a given neutron skin thickness, the slope $L$ and the $g_{\rho}$ coupling for the parametrizations with the $\sigma-\rho$ term ( $F_{\rho}$ family) is larger. For this same family, the transition density is lower and almost does not change below $L=80 \mathrm{MeV}$. As we also see, the pressure behaves differently.

It is important to look into the bulk properties of finite nuclei obtained for each of the families. Table II contains the total energy, the charge radii, the neutron radii, and the neutron skin thickness for three isospin asymmetric spherical nuclei ${ }^{48} \mathrm{Ca},{ }^{132} \mathrm{Sn}$, and ${ }^{208} \mathrm{~Pb}$, for selected parametrizations of the $F_{\rho}$ and $F_{2 \rho}$ families. The parameter sets are so chosen that they predict similar neutron skin thicknesses in ${ }^{208} \mathrm{~Pb}$ for both families. The differences between the charge radii for these nuclei obtained for the two selected models within each family are equal to or smaller than $0.015 \mathrm{fm}$ for the family $F_{\rho}$ and $0.011 \mathrm{fm}$ for the family $F_{2 \rho}$. For similar values of $\Delta r_{\mathrm{np}}$, the charge radii for the $F_{\rho}$ family are larger by $\sim 0.01 \mathrm{fm}$ than the ones obtained for the $F_{2 \rho}$ family. These differences can be leveled off by increasing the saturation density $\sim 0.001 \mathrm{fm}^{-3}$ in the case of the $F_{\rho}$ family. However, such fine tuning may not explain the observed trend of the transition density that it stays almost unchanged below $\Delta r_{\mathrm{np}}=0.22 \mathrm{fm}$ for the $F_{\rho}$ family. The values of charge radii for the $F_{\rho}$ and $F_{2 \rho}$ families are within the prediction of other RMF parametrizations that have been tuned to nuclear properties [43-45] and are employed for the study of correlations of $\Delta r_{\mathrm{np}}$ with various bulk properties of nuclear matter and the neutron stars [10,40].

In Fig. 2, we plot for both families the symmetry energy and its derivatives with respect to the density as a function of the density at subsaturation. It is clearly seen that the constraint imposed on the neutron skin leads to the crossing of the curves obtained with the different parametrizations of the $F_{\rho}$ family for the symmetry energy and its slope, respectively, below $0.1 \mathrm{fm}^{-3}$ and below $0.04 \mathrm{fm}^{-3}$, while for the $F_{2 \rho}$ family these crossings occur above those densities. In particular, it is seen that $K_{\text {sym }}$ is changing much slower at low densities within the $F_{2 \rho}$ family. These properties give rise to a different behavior of the spinodals; see Fig. 3. While for the $F_{2 \rho}$ family, the spinodal regions are larger, when the slope, $L$, is smaller, a behavior discussed in Ref. [26], the contrary occurs with the $F_{\rho}$ family. The effect of $L$ on the EOS of $\beta$-equilibrium matter
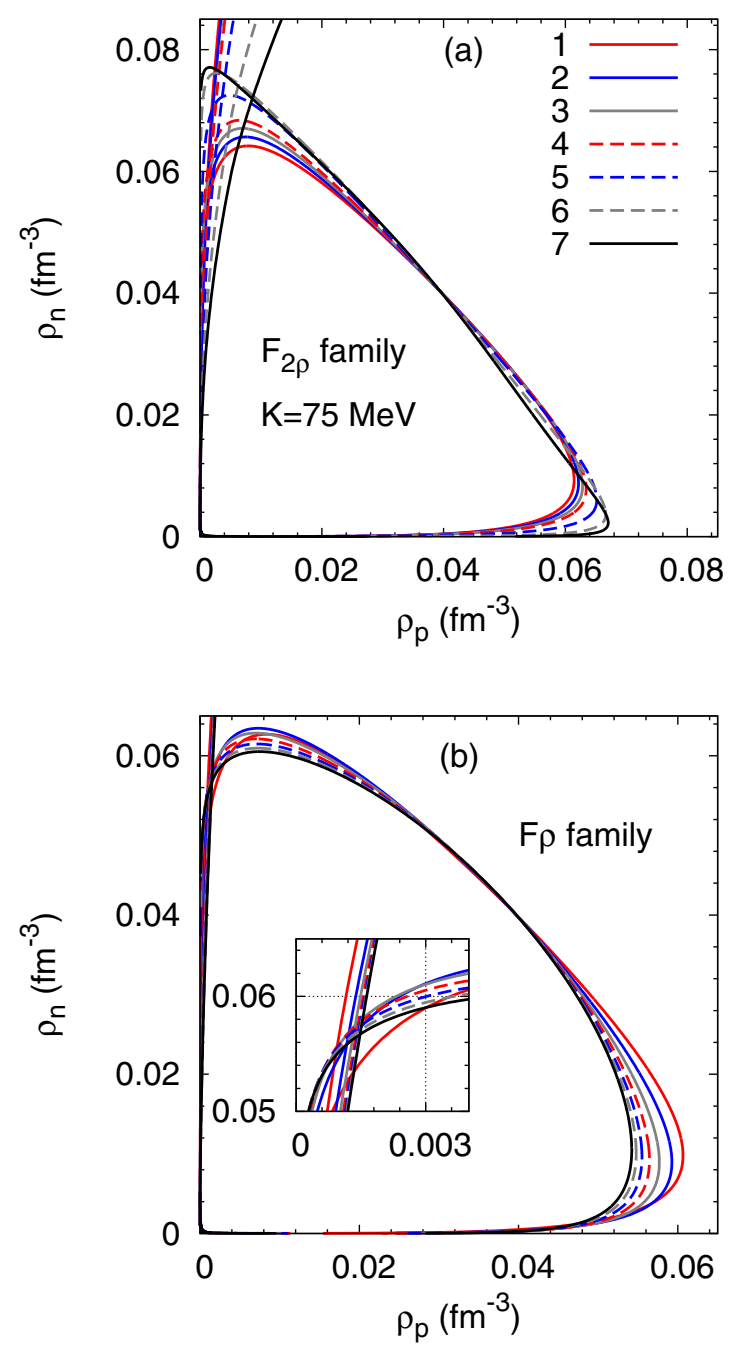

FIG. 3. Spinodal sections for the (a) $F_{2 \rho}$ and (b) $F_{\rho}$ families. The EOS of $\beta$-equilibrium stellar matter are also represented.

is the same: the smaller $L$, the larger the proton fraction for a given density. These two effects add up for the $F_{2 \rho}$ family and favor a larger transition density, while for the $F_{\rho}$ family, the two effects act in opposite directions, and, as a result, the transition density does not change much. Let us point out that the behavior of the scalar density is purely relativistic and, therefore, Skyrme forces behave all as the $F_{2 \rho}$ family and not as the $F_{\rho}$ one. Most of the RMF models describe their isovector channel through the $\rho$ meson alone, which brings a baryonic density dependence on the energy and pressure, similar to the one found with Skyrme interactions.

In Fig. 4, we show the transition pressure $P_{t}$ as a function of the slope of the symmetry energy, $L$ (left-hand panels), and as a function of the neutron skin thickness $\Delta r_{\text {np }}$ (right-hand panels), for both families considered in this study. In general, the predicted $P_{t}$ depends significantly not only on the isovector but also on the isoscalar mixing nonlinear terms used in the model. As discussed previously in Refs. [25,26], the behavior of $P_{t}$ is not monotonic with $L$. For lower $L$ values, the pressure increases with $L$, followed by a steep decrease, for the larger $L$ values. For the $F_{\rho}$ family, the pressure increases steadily, 

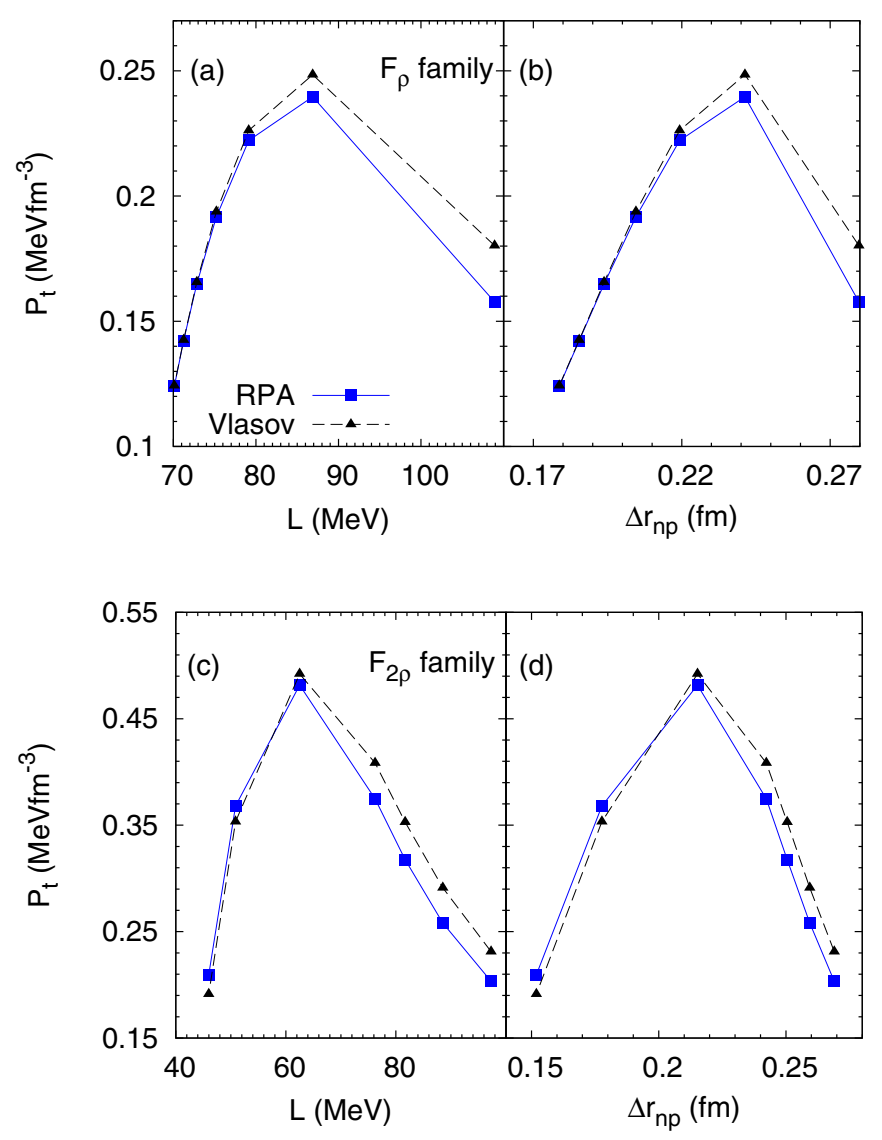

FIG. 4. Crust-core transition pressures $P_{t}$ for the (a) and (b) $F_{\rho}$ family and (c) and (d) $F_{2 \rho}$ family as a function of $L$ (left) and the neutron skin thickness $\Delta r_{\mathrm{np}}$ (right) from RRPA and Vlasov calculations.

almost linearly, until $L=85 \mathrm{MeV}$. This behavior is similar to the one discussed in Ref. [46].

In Ref. [15], it was proposed that even with entrainment the crust is large enough to explain glitches, choosing a parametrization that predicts a large pressure at the crust-core transition. A density dependence of the symmetry energy similar to the one of the $F_{2 \rho}$ family was used, and transition pressures as large as 0.425 and $0.550 \mathrm{MeV} / \mathrm{fm}^{3}$ for $L \sim$ $60 \mathrm{MeV}$ and $\Delta r_{\mathrm{np}} \sim 0.20-0.22 \mathrm{fm}$, in accordance with the results of the $F_{2 \rho}$ family, were obtained. If, however, the density dependence of the symmetry energy goes as the $F_{\rho}$ family, a smaller maximum pressure, $\sim 0.25 \mathrm{MeV} / \mathrm{fm}^{3}$, for $L \sim 85 \mathrm{MeV}$ and $\Delta r_{\mathrm{np}} \sim 0.24 \mathrm{fm}$ would have been possible. In this case, the crust would have not been enough to explain the glitches.

For completeness, we plot in Fig. 5 the EOS (top panel) for neutron star matter (lines) and pure neutron matter (dotted lines) at low densities and their corresponding binding energies ( $E / A$, bottom panel) for a few $F_{\rho}$ and $F_{2 \rho}$ parameter sets. The grey region in the bottom panel is the pure neutron matter result calculated from a chiral effective field theory (see Ref. [47] for details). The solid and open circles in the top panel correspond to the $P_{t}$ calculated from RRPA with and without Coulomb contribution, respectively. It can be seen in the top panel of

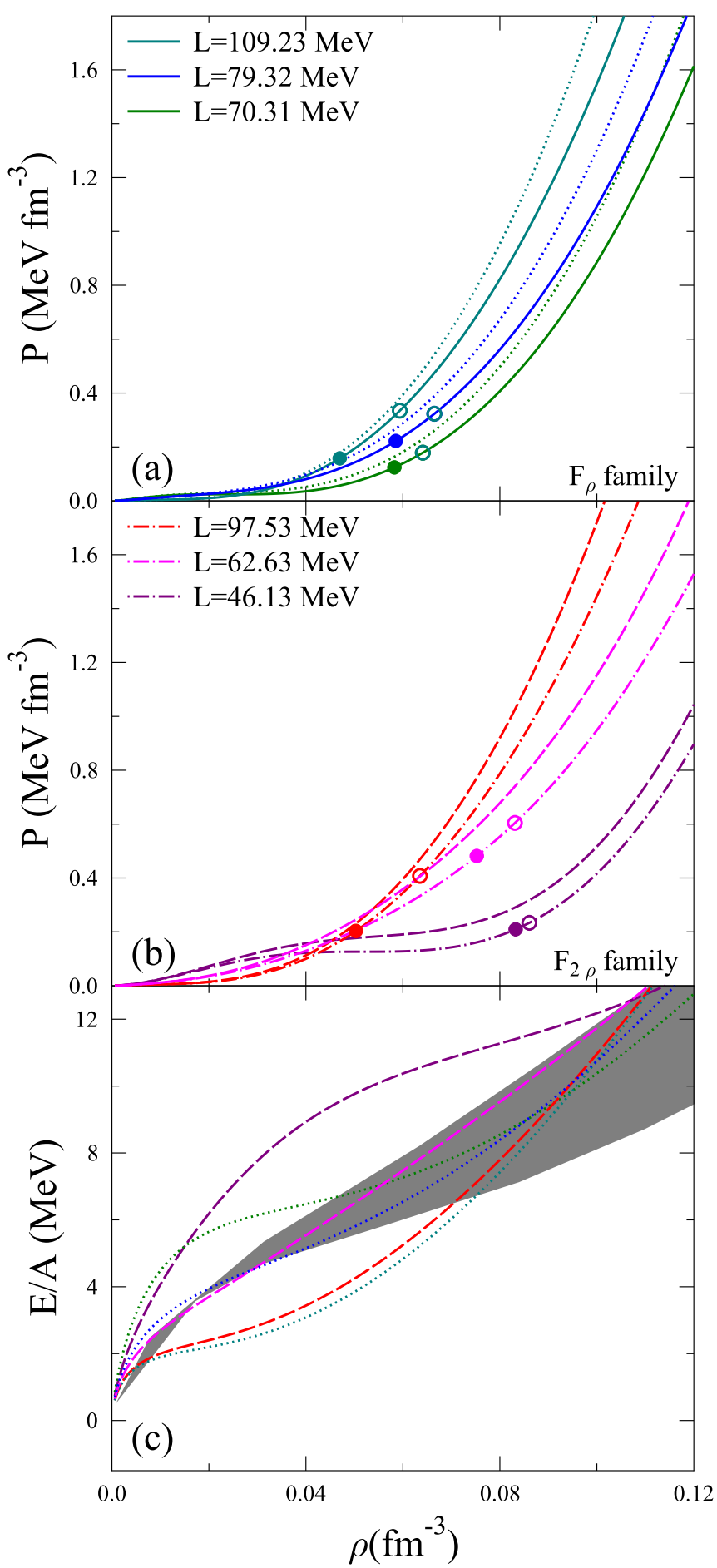

FIG. 5. EOS for the (a) $F_{\rho}$ and (b) $F_{2 \rho}$ families. The solid (dashdotted) and dotted (dashed) lines are the EOS for the $F_{\rho}\left(F_{2 \rho}\right)$ families, corresponding to NS matter and pure neutron matter, respectively. Open (solid) circles in (a) and (b) correspond to the transition pressure $P_{t}$ calculated with the RRPA method for the case without (with) the Coulomb contribution. The energy per nucleon of the pure neutron matter (c) for both families is also displayed. The grey region shown is the pure neutron matter result from Ref. [47].

Fig. 5 that the difference in $P_{t}$ appears more significantly for relatively smaller $L$. The EOS and the corresponding $E / A$ for 
NS and pure neutron matter at low densities vary significantly, depending on the parameter sets used. In general, the binding energies for pure neutron matter calculated with $F_{\rho}$ and $F_{2 \rho}$ families, with $L$ around 79.32 and $62.63 \mathrm{MeV}$, respectively, are quite compatible with the calculations from chiral effective field theory. However, the significant difference of $P_{t}$ predicted by $F_{\rho}$ and $F_{2 \rho}$ families around this range of $L$ is due to the role of the meson mixed- and self-interaction nonlinear terms in the EOS.

One consequence of the different behavior of both families could be that, contrary to what was proposed in Ref. [15], the crust might not be enough to describe glitches due to entrainment. For the two parametrizations compatible with the chiral effective theories, we have obtained 0.22 and $0.49 \mathrm{MeV} / \mathrm{fm}^{3}$ for the transition pressure for $F_{\rho}(L=79)$ and $F_{2 \rho}(L=62)$, respectively. Considering for the moment of inertia of the crust, $I_{\mathrm{cr}}$, the expression given in Refs. [15,48], with $I_{\text {cr }}$ proportional to $R_{t}^{6} P_{t} / R$, we get for a $1.4 M_{\odot}$ star, $I_{\mathrm{cr}}\left[F_{\rho}(L=79)\right] / I_{\mathrm{cr}}\left[F_{2 \rho}(L=62)\right] \sim 2 / 3$, indicating that the contribution of the crust moment of inertia may be smaller than the prediction of Ref. [15]. The radius at the crustcore transition was estimated by taking the Baym, Pethick, and Sutherland EOS [49] for the outer crust and the FSU inner crust obtained in the framework of a Thomas-Fermi calculation [50] and matching the homogeneous matter EOS to the inner crust EOS at the crust-core transition. We expect the inner crust of the FSU to be a good choice because this model has a slope $L$ similar to the two models. We obtained $R_{t}\left[F_{\rho}(L=79)\right] / R_{t}\left[F_{2 \rho}(L=62)\right] \sim 1.03$.

\section{CONCLUSIONS}

In the present work, the influence of the density dependence of the symmetry energy on properties such as the density and pressure at the crust-core transition was analyzed within two families of RMF models. We used three different methods to calculate the transition density: the thermodynamical spinodal, the dynamical spinodal within the Vlasov formalism, and the RRPA. It was shown that the last two methods give similar results, confirming previous studies [41,42]. The thermodynamical spinodal also gives a good estimate of the transition density, as already shown in Ref. [26], and involves simpler calculations. The mixed terms involving the $\rho$ meson and the $\sigma$ or the $\omega$ mesons allow the modification of the density dependence of the symmetry energy. The two families of the RMF models differ in mixed nonlinear meson terms in the isovector part of the Lagrangian density. Both families of the RMF models have the same isoscalar properties, but the isovector channel is modified through a $\sigma \rho^{2}$ term in the $F_{\rho}$ family, and a $\omega^{2} \rho^{2}$ term in the $F_{2 \rho}$ family. The parameters that describe the isovector channel were appropriately adjusted so that different neutron skin thicknesses were obtained for the ${ }^{208} \mathrm{~Pb}$ nucleus. Since the $\sigma$-meson field is proportional to the scalar density and the $\omega$-meson field to the baryonic density, different behaviors of the crust-core transition properties were observed.

The scalar density is a relativistic quantity, and by performing an expansion of the RMF energy density in powers of the Fermi momentum, it was shown in Ref. [19] that relativistic corrections coming from the Lorentz contraction factor in the scalar density have an effect equivalent to repulsive many-body forces. Owing to the much faster increase of the scalar density at low densities followed by a smoothing at larger, but still subsaturation, densities, the same neutron skin thicknesses were obtained for the $F_{\rho}$ family, with larger values of the slope $L$. Also the crust-core transition was affected. The values of the pressure at the transition are lower, there is no clear decrease of the transition density with $L$, and the transition pressure increases with the slope $L$ for $L<85 \mathrm{MeV}$. The $F_{2 \rho}$ family, however, behaves as discussed in previous works $[11,25,26]$, where both nonrelativistic and relativistic models have been considered, giving rise to similar conclusions. The behavior of the $F_{2 \rho}$ family is defined by the baryonic density and, therefore, does not contain explicit relativistic effects. If the density dependence of the symmetry energy should be defined by the scalar density, we may expect smaller pressures at the crust-core transition. In this case, the crust would probably not be enough to describe glitches if entrainment is taken into account.

\section{ACKNOWLEDGMENTS}

H.P. is supported by FCT under Project No. SFRH/BPD/95566/2013. Partial support comes from "NewCompStar," COST Action MP1304. A.S. is partly supported by the Research-Cluster-Grant Program of the University of Indonesia, under Contract No. 1862/UN.R12/HKP.05.00/2015.
[1] A. W. Steiner, M. Prakash, J. M. Lattimer, and P. J. Ellis, Phys. Rep. 411, 325 (2005).

[2] B.-A. Li, L.-W. Chen, and C.-M. Ko, Phys. Rep. 464, 113 (2008).

[3] M. B. Tsang, J. R. Stone, F. Camera, P. Danielewicz, S. Gandolfi, K. Hebeler, C. J. Horowitz, J. Lee, W. G. Lynch, Z. Kohley, R. Lemmon, P. Möller, T. Murakami, S. Riordan, X. Roca-Maza, F. Sammarruca, A. W. Steiner, I. Vidaña, and S. J. Yennello, Phys. Rev. C 86, 015803 (2012).

[4] U. Garg, T. Li, S. Okumura, H. Akimune, M. Fujiwara, M. Harakeh, H. Hashimoto, M. Itoh, Y. Iwao, T. Kawabata, K. Kawase, Y. Liu, R. Marks, T. Murakami, K. Nakanishi, B. Nayak, P. M. Rao, H. Sakaguchi, Y. Terashima, M. Uchida,
Y. Yasuda, M. Yosoi, and J. Zenihirof, Nucl. Phys. A 788, 36 (2007).

[5] P. Danielewicz and J. Lee, Nucl. Phys. A 818, 36 (2009).

[6] B.-A. Li, G.-C. Yong, and W. Zuo, Phys. Rev. C 71, 014608 (2005).

[7] C. Fuchs, Prog. Part. Nucl. Phys. 56, 1 (2006).

[8] B. A. Brown, Phys. Rev. Lett. 85, 5296 (2000).

[9] S. Typel and B. A. Brown, Phys. Rev. C 64, 027302 (2001).

[10] C. J. Horowitz and J. Piekarewicz, Phys. Rev. Lett. 86, 5647 (2001).

[11] I. Vidaña, C. Providência, A. Polls, and A. Rios, Phys. Rev. C 80, 045806 (2009). 
[12] B. Link, R. I. Epstein, and J. M. Lattimer, Phys. Rev. Lett. 83, 3362 (1999).

[13] N. Chamel, Phys. Rev. Lett. 110, 011101 (2013).

[14] N. Andersson, K. Glampedakis, W. C. G. Ho, and C. M. Espinoza, Phys. Rev. Lett. 109, 241103 (2012).

[15] J. Piekarewicz, F. J. Fattoyev, and C. J. Horowitz, Phys. Rev. C 90, 015803 (2014).

[16] H. Müller and B. D. Serot, Nucl. Phys. A 606, 508 (1996).

[17] S. Typel and H. H. Wolter, Nucl. Phys. A 656, 331 (1999).

[18] B. K. Agrawal, Phys. Rev. C 81, 034323 (2010).

[19] B. D. Serot and J. D. Walecka, Int. J. Mod. Phys. E 6, 515 (1997).

[20] C. Providência, L. Brito, A. M. S. Santos, D. P. Menezes, and S. S. Avancini, Phys. Rev. C 74, 045802 (2006).

[21] B. K. Agrawal, A. Sulaksono, and P. Reinhard, Nucl. Phys. A 882, 1 (2012).

[22] A. Sulaksono and T. Mart, Phys. Rev. C 74, 045806 (2006).

[23] H. Pais, A. Santos, L. Brito, and C. Providência, Phys. Rev. C 82, 025801 (2010).

[24] S. S. Avancini, S. Chiacchiera, D. P. Menezes, and C. Providência, Phys. Rev. C 82, 055807 (2010).

[25] C. Ducoin, C. Providência, A. M. Santos, L. Brito, and P. Chomaz, Phys. Rev. C 78, 055801 (2008).

[26] C. Ducoin, J. Margueron, C. Providência, and I. Vidaña, Phys. Rev. C 83, 045810 (2011).

[27] J. D. Walecka, Ann. Phys. 83, 491 (1974).

[28] R. J. Furnstahl, Nucl. Phys. A 706, 85 (2002).

[29] T. Sil, M. Centelles, X. Viñas, and J. Piekarewicz, Phys. Rev. C 71, 045502 (2005).

[30] S. K. Dhiman, R. Kumar, and B. K. Agrawal, Phys. Rev. C 76, 045801 (2007).

[31] N. Alam, A. Sulaksono, and B. K. Agrawal, Phys. Rev. C 92, 015804 (2015).
[32] S. Kubis, Phys. Rev. C 70, 065804 (2004).

[33] S. Kubis, Phys. Rev. C 76, 025801 (2007).

[34] J. M. Lattimer and M. Prakash, Phys. Rep. 442, 109 (2007).

[35] M. Nielsen, C. Providência, and J. da Providência, Phys. Rev. C 44, 209 (1991).

[36] M. Nielsen, C. Providência, and J. da Providência, Phys. Rev. C 47, 200 (1993).

[37] S. S. Avancini, L. Brito, D. P. Menezes, and C. Providência, Phys. Rev. C 71, 044323 (2005).

[38] H. Pais and C. Providência (unpublished).

[39] C. Providência, L. Brito, S. S. Avancini, D. P. Menezes, and P. Chomaz, Phys. Rev. C 73, 025805 (2006).

[40] J. Carriere, C. J. Horowitz, and J. Piekarewicz, Astrophys. J. 593, 463 (2003).

[41] C. Ducoin, J. Margueron, and P. Chomaz, Nucl. Phys. A 809, 30 (2008).

[42] C. J. Horowitz and G. Shen, Phys. Rev. C 78, 015801 (2008).

[43] Y. Sugahara and H. Toki, Nucl. Phys. A 579, 557 (1994).

[44] B. G. Todd-Rutel and J. Piekarewicz, Phys. Rev. Lett. 95, 122501 (2005).

[45] G. A. Lalazissis, J. König, and P. Ring, Phys. Rev. C 55, 540 (1997).

[46] C. C. Moustakidis, T. Niksic, G. A. Lalazissis, D. Vretenar, and P. Ring, Phys. Rev. C 81, 065803 (2010).

[47] T. Krüger, I. Tews, K. Hebeler, and A. Schwenk, Phys. Rev. C 88, 025802 (2013).

[48] F. J. Fattoyev and J. Piekarewicz, Phys. Rev. C 82, 025810 (2010).

[49] G. Baym, C. Pethick, and P. Sutherland, Astrophys. J. 170, 299 (1971).

[50] F. Grill, H. Pais, C. Providência, I. Vidaña, and S. S. Avancini, Phys. Rev. C 90, 045803 (2014). 